\title{
Peak-to-Average Power Ratio Reduction in Orthogonal Frequency Division Multiplexing Systems
}

\author{
Pooria Varahram and Borhanuddin Mohd Ali \\ Universiti Putra Malaysia, \\ Malaysia
}

\section{Introduction}

Broadband wireless is a technology that provides connection over the air at high speeds. Orthogonal frequency division multiplexing (OFDM) system has generally been adopted in recent mobile communication systems because of its high spectral efficiency and robustness against intersymbol interference (ISI). However, due to the nature of inverse fast Fourier transform (IFFT) in which the constructive and destructive behaviour could create high peak signal in constructive behaviour while the average can become zero at destructive behaviour, OFDM signals generally become prone to high peak-to-average power ratio (PAPR) problem. In this chapter, we focus on some of the techniques to overcome the PAPR problem (Krongold and Jones, 2003; Bauml, et al. 1996).

The other issue in wireless broadband is how to maximize the power efficiency of the power amplifier. This can be resolved by applying digital predistortion to the power amplifier (PA) (Varahram, et al. 2009). High PAPR signal when transmitted through a nonlinear PA creates spectral broadening and increase the dynamic range requirement of the digital to analog converter (DAC). This results in an increase in the cost of the system and a reduction in efficiency. To address this problem, many techniques for reducing PAPR have been proposed. Some of the most important techniques are clipping (Kwon, et al. 2009), windowing (Van Nee and De Wild, 1998), envelope scaling (Foomooljareon and Fernando, 2002), random phase updating (Nikookar and Lidsheim, 2002), peak reduction carrier (Tan and Wassell, 2003), companding (Hao and Liaw, 2008), coding (Wilkison and Jones, 1995), selected mapping (SLM) (Bauml, et al. 1996), partial transmit sequence (PTS) (Muller and Huber, 1997), DSI-PTS (Varahram et al. 2010), interleaving (Jayalath and Tellambura, 2000), active constellation extension (ACE) (Krongold, et al. 2003), tone injection and tone reservation (Tellado, 2000), dummy signal insertion (DSI) (Ryu, et al. 2004), addition of Guassian signals (Al-Azoo et al. 2008) and etc (Qian, 2005).

Clipping is the simplest technique for PAPR reduction, where the signal at the transmitter is clipped to a desired level without modifying the phase information. In windowing a peak of the signal is multiplied with a part of the frame. This frame can be 
in Gaussian shape, cosine, Kaiser or Hanning window, respectively. In companding method the OFDM signal is companded before digital to analog conversion. The OFDM signal after IFFT is first companded and quantized and then transmitted through the channel after digital to analog conversion. The receiver first converts the signal into digital format and then expands it. The companding method has application in speech processing where high peaks occur infrequently. In PTS, by partitioning the input signal and applying several IFFT, the optimum phase sequence with lowest PAPR will be selected before being transmitted. This technique results in high complexity. In SLM, a copy of input signal is used to choose the minimum PAPR among the multiple signals. We can conclude that there is always a trade-off in choosing a particular PAPR technique. The trade-off comes in the form of complexity, power amplifier output distortion, cost, side information, PAPR reduction, Bit Error Rate (BER) performance, spectrum efficiency and data rate loss.

\section{OFDM signal}

In OFDM systems, first a specific number of input data samples are modulated (e.g. PSK or QAM), and by IFFT technique the input samples become orthogonal and will be converted to time domain at the transmitter side. The IFFT is applied to produce orthogonal data subcarriers. In theory, IFFT combines all the input signals (superposition process) to produce each element (signal) of the output OFDM symbol. The time domain complex baseband OFDM signal can be represented as (Han and Lee, 2005):

$$
x_{n}=\frac{1}{\sqrt{N}} \sum_{k=0}^{N-1} X k e^{j 2 \pi \frac{n}{N} k}, \quad n=0,1,2, \ldots \ldots ., N-1
$$

where $x_{n}$ is the $\mathrm{n}$-th signal component in OFDM output symbol, $X_{k}$ is the $k$-th data modulated symbol in OFDM frequency domain, and $N$ is the number of subcarrier.

The PAPR of the transmitted OFDM signal can be given by (Cimini and Sollenberger, 2000):

$$
\operatorname{PAPR}(d B)=\frac{\max \left[\left|x_{n}\right|^{2}\right]}{E\left[\left|x_{n}\right|^{2}\right]}
$$

where $E[$.$] is the expectation value operator. The theoretical maximum of PAPR for N$ number of subcarriers is as follows:

$$
P A P R_{\max }=10 \log (N) d B
$$

PAPR is a random variable since it is a function of the input data, while the input data is a random variable. Therefore PAPR can be analyzed by using level crossing rate theorem which calculates the mean number of times that the envelope of a stationary signal crosses a 
given level. Knowing the amplitude distribution of the OFDM output signals, it is easy to compute the probability that the instantaneous amplitude will lie above a given threshold and the same goes for power. This is performed by calculating the complementary cumulative distribution function (CCDF) for different PAPR values as follows:

$$
C C D F=\operatorname{Pr}\left(P A P R>P A P R_{0}\right)
$$

Here the effect of additive white Gaussian noise (AWGN) on OFDM performance is studied. As OFDM systems use standard digital modulation formats to modulate the subcarriers, PSK and QAM are usually used due to their excellent error resilient properties. The most important block in OFDM is IFFT. IFFT changes the distribution of the signal without altering its average power. The BER or bit error probability $P_{b e}$ in an AWGN channel is given by (Han and Lee, 2005):

$$
P_{b e, M Q A M} \approx \frac{4(\sqrt{M}-1)}{k \sqrt{M}} Q\left(\sqrt{\frac{3 k}{(M-1)} \cdot \frac{E_{b}}{N_{o}}}\right)
$$

where $M$ is the modulation order, $k=\log _{2}(M)$ is the number of bits per symbol, and $Q($.$) is$ the Gaussian $Q$ function defined as:

$$
Q(y)=\operatorname{erfc}\left(\frac{y}{\sqrt{2}}\right)
$$

In this chapter the performance of BER versus energy per bit to noise power spectral density ratio $\left(E_{\mathrm{b}} / N_{\mathrm{o}}\right)$ is analyzed.

\section{PAPR reduction techniques}

In this section, some of the most important PAPR reduction techniques such as Selected Mapping (SLM), Partial Transmit Sequence (PTS) and Enhanced PTS EPTS) are presented.

\subsection{Conventional SLM (C-SLM)}

In Conventional SLM (C-SLM) method, OFDM signal is first converted from serial to parallel by means of serial-to-parallel converter. The parallel OFDM signal is then multiplied by several phase sequences that are created offline and stored in a matrix. A copy of the OFDM signal is multiplied with a random vector of phase sequence matrix. For each subblock IFFT is performed and its PAPR is calculated to look for the minimum one. The OFDM signal having minimum PAPR is then selected and be transmitted. The main drawbacks of this technique are the high complexity due to the high number of subblocks and the need to send side information which result in data rate and transmission efficiency degradation, respectively. In Fig. 1, the number of candidate signal or subblocks is given by $U$, hence $\log _{2} U$ number of bits is required to be sent as side information.

The other drawback of this method is that by increasing $U$, higher number of IFFT blocks are required which increase the complexity significantly. Hence, a method with low complexity and high PAPR performance is required. 


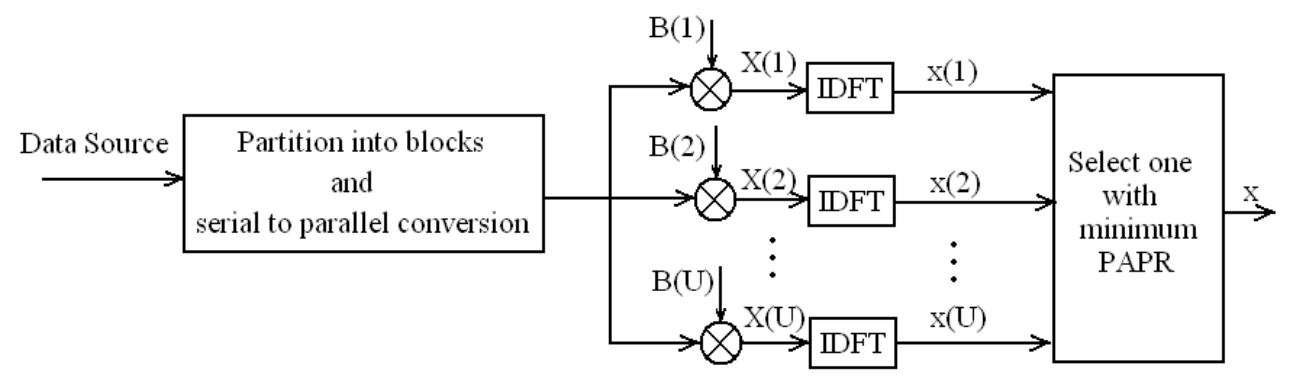

Fig. 1. The block diagram of the C-SLM method.

\subsection{Conventional PTS (C-PTS)}

To analyze C-PTS let $\mathrm{X}$ denotes random input signal in frequency domain with length $N$. $X$ is partitioned into $V$ disjoint subblocks $X_{v}=\left[X_{v, 0}, X_{v, 1}, \ldots, X_{v, N-1}\right]^{T}, v=1,2, \ldots, V$ such that $\sum_{v=1}^{V} X_{v}=X$ and then these subblocks are combined to minimize the PAPR in time domain.

The Sbblock partitioning is based on interleaving in which the computational complexity is less compared to adjacent and pseudo-random, however it gives the worst PAPR performance among them (Han and Lee, 2005).

By applying the phase rotation factor $b_{v}=e^{j \phi_{v}}, v=1,2, \ldots, V$ to the IFFT of the $v$ th subblock $X_{\mathrm{v}}$, the time domain signal after combining is obtained as:

$$
x^{\prime}(b)=\sum_{v=1}^{V} b_{v} x_{v}
$$

where $x^{\prime}(b)=\left[x_{0}^{\prime}(b), x_{1}^{\prime}(b), \ldots x_{N F-1}^{\prime}(b)\right]^{T}$. The objective is to find the optimum signal $x^{\prime}(b)$ with the lowest PAPR.

Both $\mathrm{b}$ and $\mathrm{x}$ can be shown in matrix forms as follows:

$$
\begin{aligned}
& b=\left[\begin{array}{ccc}
b_{1}, & b_{1}, \ldots, b_{1} \\
\vdots & \vdots & \vdots \\
b_{V}, & b_{V}, \ldots, & b_{V}
\end{array}\right]_{V \times N} \\
& x=\left[\begin{array}{ccc}
x_{1,0}, & x_{1,1}, \ldots, & x_{1, N F-1} \\
\vdots & \vdots & \vdots \\
x_{V, 0}, & x_{V, 1}, \ldots, & x_{V, N F-1}
\end{array}\right]_{V \times N F}
\end{aligned}
$$

Fig. 2 shows the block diagram of C-PTS. It should be noted that all the elements of each row of matrix $b$ are of the same values and this is in accordance with the C-PTS method. In order to obtain exact PAPR calculation, at least four times oversampling is necessary (Han and Lee, 2005). 


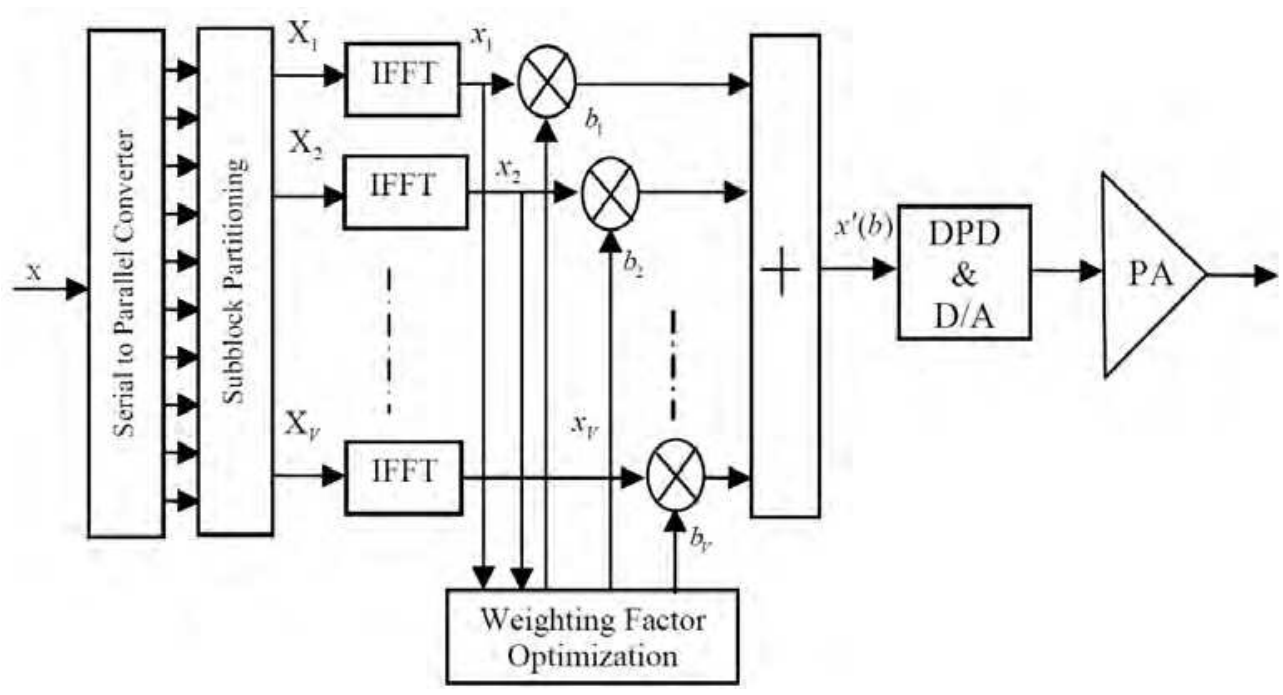

Fig. 2. Block diagram of the C-PTS scheme with Digital predistortion and power amplifier in series

This process is performed by choosing the optimization parameter $\tilde{b}$ which satisfies the following condition:

$$
\tilde{b}=\operatorname{argmin}\left(\max _{0 \leq k \leq N F-1}\left|\sum_{v=1}^{V} b_{v} x_{v}\right|\right)
$$

where $V$ is the number of subblocks partitioning and $F$ is the oversampling factor. After obtaining the optimum $\tilde{b}$, the signal is transmitted.

For finding the optimum $\tilde{b}$, we should perform exhaustive search for $(V-1)$ phase factors since one phase factor can remain fixed, $b_{1}=1$. Hence to find the optimum phase factor, $W V-1$ iteration should be performed, where $W$ is the number of allowed phase factors.

\subsection{Enhanced PTS (EPTS)}

In order to decrease the complexity of C-PTS, a new phase sequence is generated. The block diagram of the enhanced partial transmit sequence (EPTS) scheme is shown in Fig. 3.

This new phase sequence is based on the generation of $N$ random values of $\{1-1 j-j\}$ if the allowed phase factors is $W=4$. The phase sequence matrix can be given by: 


$$
\hat{b}=\left[\begin{array}{ccc}
b_{1,1} & , \ldots, & b_{1, N} \\
\vdots & \vdots & \vdots \\
b_{V, 1} & , \ldots, & b_{2, N} \\
b_{V+1,1} & , \ldots, & b_{V+1, N} \\
\vdots & \vdots & \vdots \\
b_{P, 1} & , \ldots, & b_{P, N}
\end{array}\right]_{[P \times N]}
$$

where $P$ is the number of iterations that should be set in accordance with the number of iterations of the C-PTS and $N$ is the number of samples (IFFT length) and $V$ is the number of subblock partitioning. The value of $\mathrm{P}$ is given as follows:

$$
P=D W^{V-1} \quad, \quad D=1,2, \ldots, D_{N}
$$

where $D$ is the coefficient that can be specified based on the PAPR reduction and complexity requirement and $D_{N}$ is specified by the user. The value of $P$ explicitly depends on the number of subblocks $V$, if the number of allowed phase factor remains constant.

There is a tradeoff for choosing the value of $D$. higher $D$ leads to higher PAPR reduction but at the expense of higher complexity; while lower $D$ results in smaller PAPR reduction but with less complexity. For example if $W=2$ and $V=4$, then in C-PTS there are 8 iterations and hence $P=8 D$. If $D=2$, then $P=16$ and both methods have the same number of iterations. But when $D=1$, then number of iterations to find the optimum phase factor will be reduced to 4 and this will result in complexity reduction. The main advantage of this method over C-PTS is the reduction of complexity while at the same time maintaining the same PAPR performance. In the case of CPTS, each row of the matrix $\hat{b}$ contains same phase sequence while each column is periodical with period $V$, whereas in the proposed method each element of matrix $\hat{b}$ has different random values. The other formats that matrix in (11) can be expressed are as follows:

$$
\begin{aligned}
& \hat{b}=\left[\begin{array}{lcc}
\overbrace{b_{1,1}, \ldots,} b_{1, N / P} & , \ldots, & b_{1,1}, \ldots, b_{1, N / P} \\
\vdots & \vdots & \vdots \\
b_{V, 1}, \ldots, b_{V, N / P} & , \ldots, & b_{V, 1}, \ldots, b_{V, N / P} \\
b_{V+1,1}, \ldots, b_{V+1, N / P}, \ldots, & b_{V+1,1}, \ldots, b_{V+1, N / P} \\
\vdots & \vdots & \vdots \\
b_{P, 1}, \ldots, b_{P, N / P} & , \ldots, & b_{P, 1}, \ldots, b_{P, N / P}
\end{array}\right]_{[P \times N]}
\end{aligned}
$$

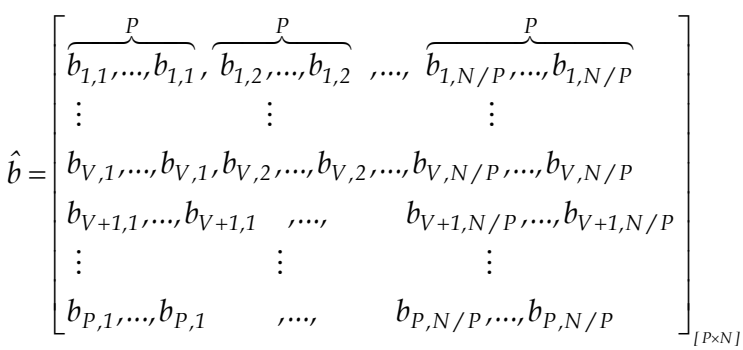


where (13) and (14) are the interleaved and adjacent phase sequences matrix, respectively.

As an example take the case of $N=256$, and the number of allowed phase factor and subblock partitioning are $W=4$ and $V=4$ respectively. With C-PTS there are $W^{M-1}=64$ possible iterations, whereas for the proposed method, in the case of $D=2$, the phase sequence is a matrix of [128x256] elements according to (11). In this case 64 iterations are required for finding the optimum phase sequence, because each two rows of the matrix in (11) multiply point-wise with the time domain input signal $x_{v}$ with length [2x256].

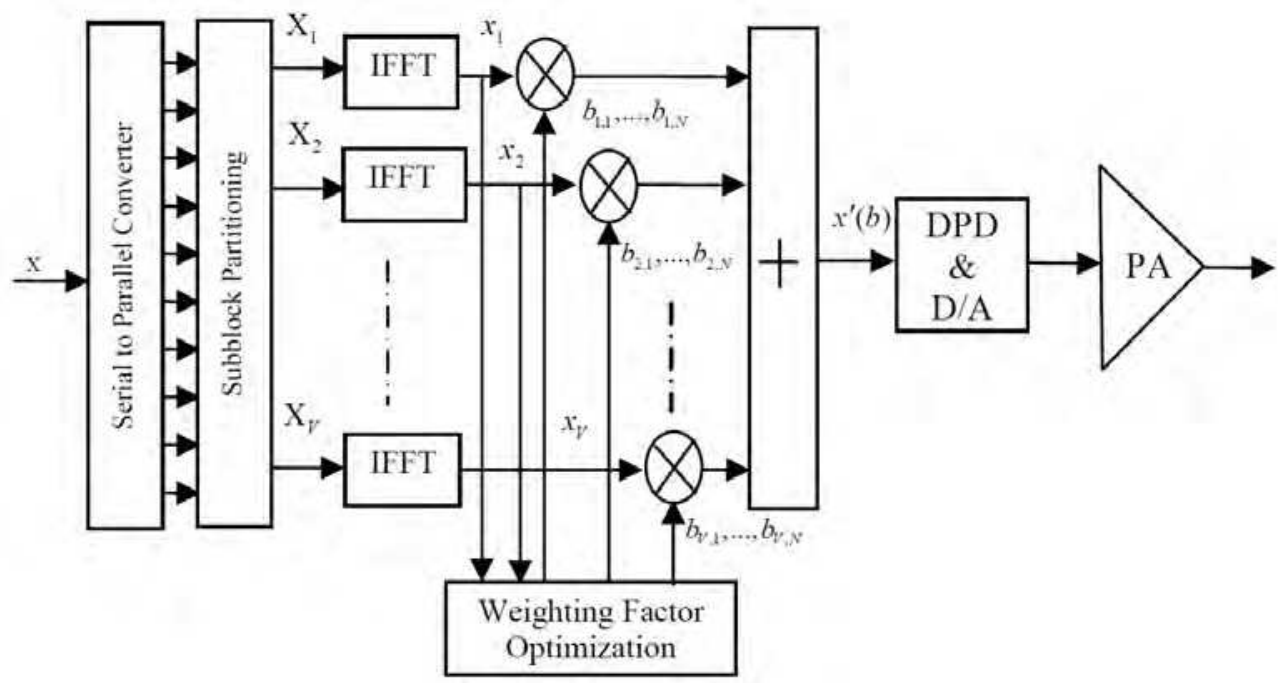

Fig. 3. The block diagram of enhanced PTS

The reduction of subblocks to 2 is because it gives almost the same PAPR reduction as CPTS with $V=4$. It should be noted that if $D=1$ then the complexity increases while if $D>2$ then the PAPR reduction is less.

Therefore the algorithm can be expressed as follows:

Step 1: Generate the input data stream and map it to the M-QAM modulation.

Step 2: Construct a matrix of random phase sequence with dimension of [PxN].

Step 3: Point-wise multiply signal $x_{v}$ with the new phase sequence.

Step 4: Find the optimum phase sequence after $P$ iterations to minimize the PAPR. 


\subsubsection{Numerical analysis}

In order to evaluate and compare the performance of the PAPR methods with C-PTS, simulations have been performed. In all the simulations, we employed QPSK modulation with IFFT length of $N=512$, and oversampling factor $F=4$. To obtain the complementary cumulative distribution function (CCDF), 40000 random OFDM symbols are generated.

Fig. 4 shows the CCDF of three different types of phase sequences interleaved, adjacent and random for $\mathrm{D}=2$. From this figure, $\mathrm{PAPR}$ reduction with random phase sequence outperforms the other types and hence this type of phase sequence is applied in the following simulations.

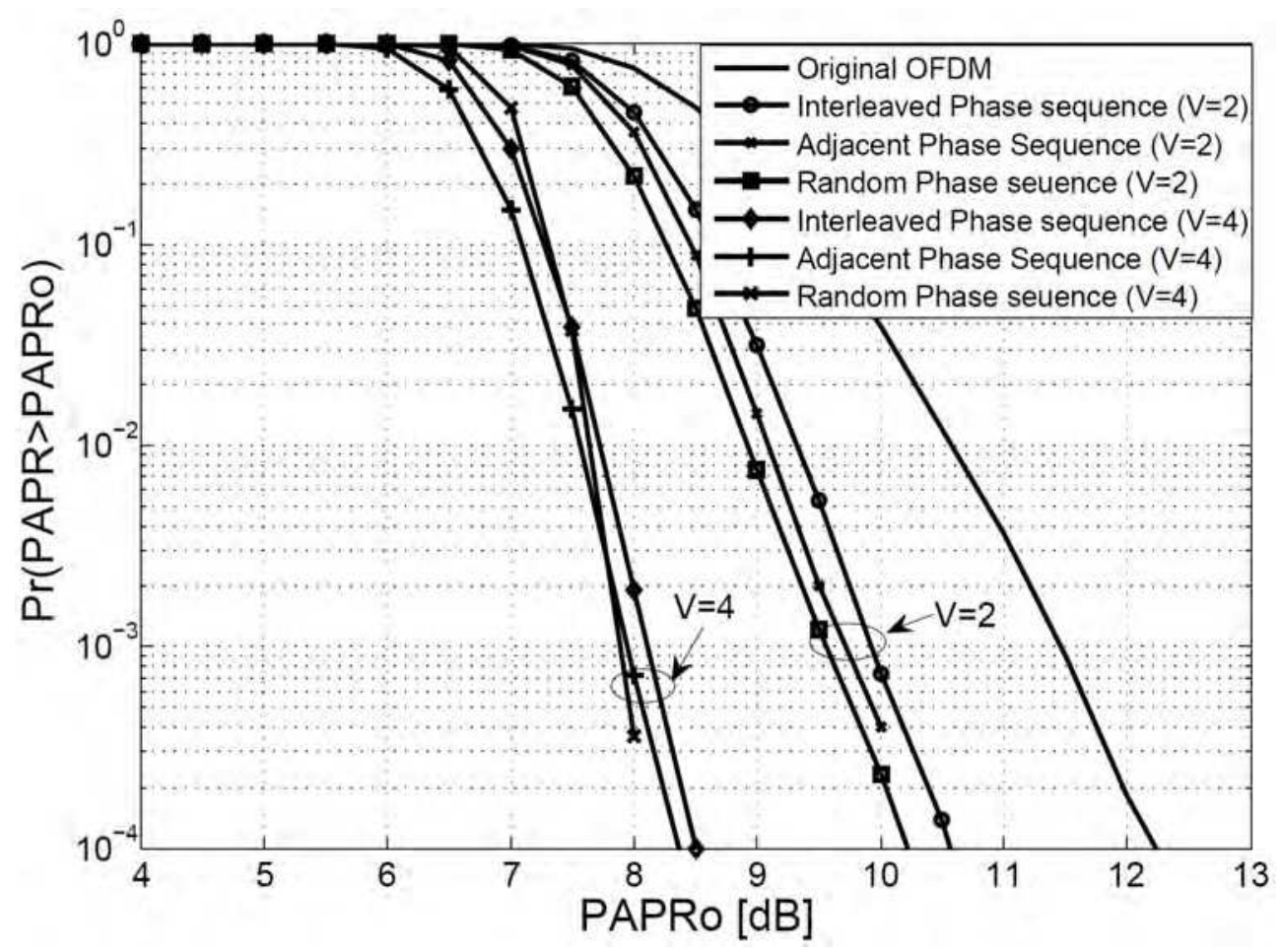

Fig. 4. CCDF of PAPR of the proposed method for different phase sequence when $D=2$

Fig. 5 shows the CCDF comparison of the PAPR of the C-PTS and EPTS for V=2 and 4 . It is clear that the proposed EPTS shows better PAPR performance compared to C-PTS where almost $0.3 \mathrm{~dB}$ reduction is achieved with EPTS. 


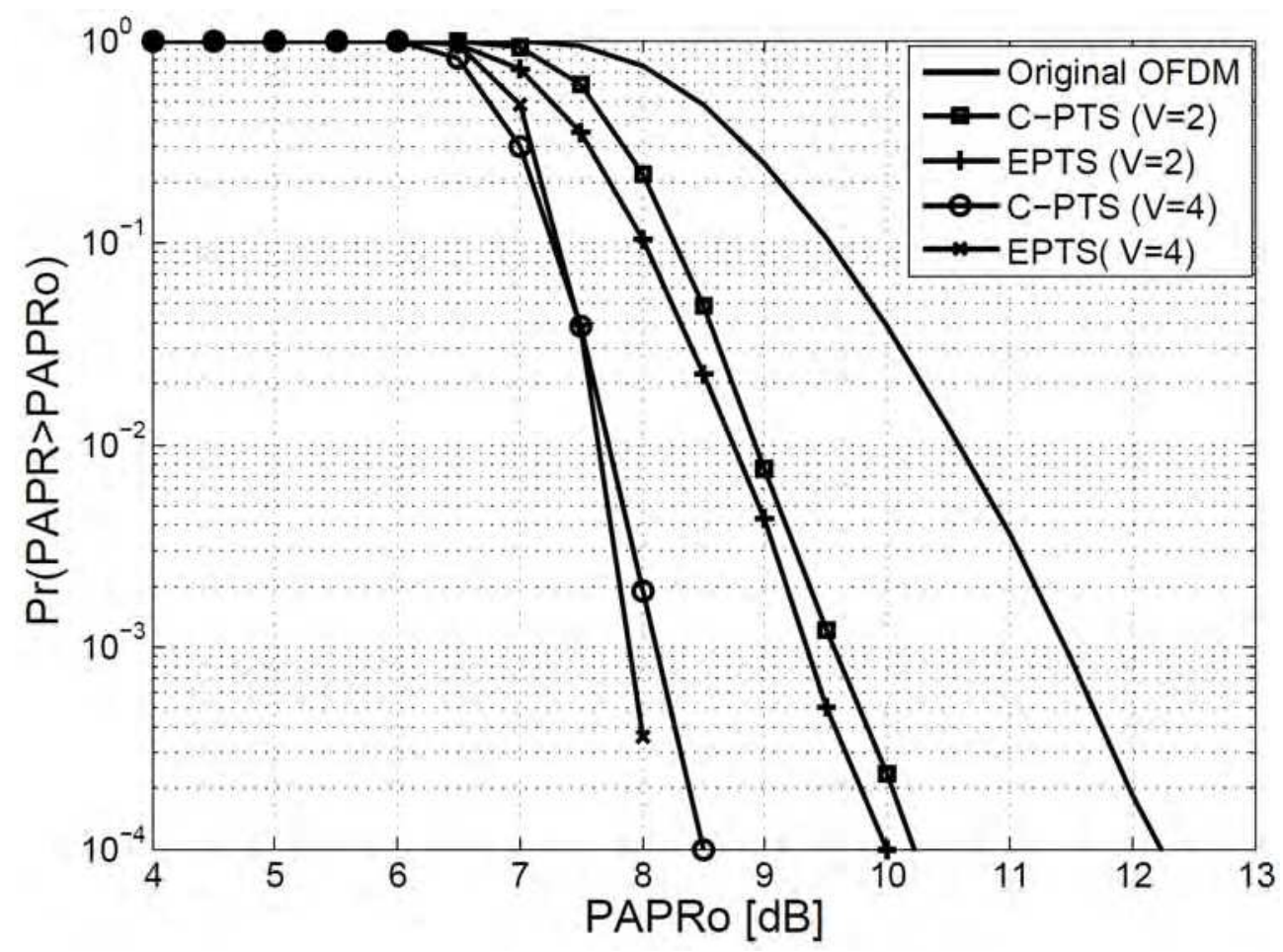

Fig. 5. CCDF comparison of PAPR of the proposed EPTS and C-PTS

\subsection{Dummy Sequence Insertion (DSI)}

The DSI method reduces PAPR by increasing the average power of the signal. Here, after converting the input data stream into parallel through the serial to parallel converter a, dummy sequence is inserted in the input signal. Therefore, the average value in Equation (2) is increased and the PAPR is subsequently reduced (Ryu, et al. 2004). IEEE 802.16d standard, specifies that the data frame of OFDM signal is allocated with 256 subcarriers which is composed of 192 data subcarriers, 1 zero DC subcarrier, 8 pilot subcarriers, and 55 guard subcarriers. Therefore, the dummy sequence can be inserted within the slot of 55 guard subcarriers without degradation of user data. However, if added dummies are more than 55, the length of the data and the bandwidth required, will be increased. This will degrade the Transmission Efficiency (TE) which is defined as:

$$
T E=\frac{K}{K+L} \times 100 \%
$$

where $K$ is the number of the subcarriers and $L$ is the number of dummy sequence. In this chapter we apply a different DSI method from the one in (Ryu, et al. 2004), where the TE is always $100 \%$. 


\subsection{Dummy Sequence Insertion with Partial Transmit Sequence (DSI-PTS)}

The block diagram of this technique is shown in Fig. 6. A complex valued dummy signals are first generated and then added to the vector of data subcarriers. The new vector in frequency domain is then constructed from K-data and L-dummy subcarriers, respectively. $\mathrm{L}$ can be any number less than $\mathrm{K}$. The new vector $\mathrm{S}$ is given by:

$$
S=\left[X_{k}, W_{l}\right]
$$

where $X_{k}=\left[X_{k, 0}, X_{k, 1}, \ldots, X_{k, N-L-1}\right], k=1,2, \ldots, K$ is the data subcarrier vector and $W_{l}=\left[W_{l, 0}, W_{l, 1}, \ldots, W_{l, L-1}\right], l=1,2, \ldots, L$ is the dummy signals vector.

After generation of the optimum OFDM signal then the PAPR is checked with the acceptable threshold that was pre-defined before. If the PAPR value is less than the threshold then the OFDM signal will be transmitted otherwise the dummy sequence is generated again as depicted with the feedback in Fig. 6 . This process is one iteration. The number of iterations can be increased to achieve the desired PAPR ( $\left.P A P R_{t h}\right)$ reduction but the processing time will also increase likewise and causes the system performance to drop.

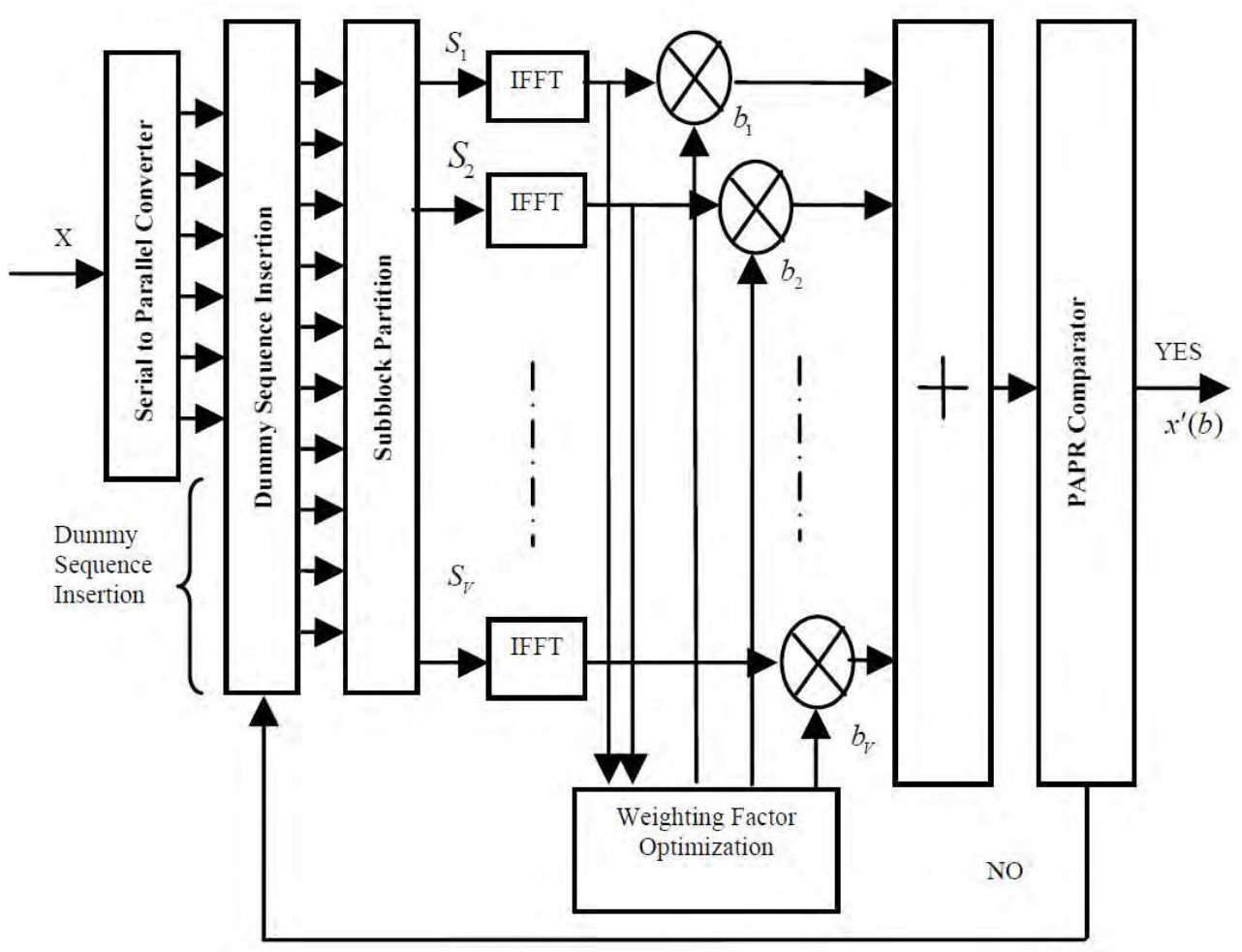

Fig. 6. Block diagram of DSI-PTS technique 
As for the DSI-PTS method, consider $L$ as the number of dummy sequence which later will be shown to be $L \leq 55$ and $N$ is the IFFT length which is 256 in the case of fixed WiMAX that includes 192 data carriers, 8 pilots and 55 zero padding and 1 dc subcarrier. Here complementary sequence is applied for the DSI (Ryu, et al. 2004).

From the block diagram in Fig. $6, X$ is the input signal stream with length $N$ after which the dummy sequence is added. The dummy sequence can be replaced with zeros in data sample. This makes the IFFT length remain unchanged and decoding of the samples in receiver becomes simpler. Then the signal is partitioned into $V$ disjoint blocks

$$
S_{v}=\left[S_{1}, S_{2}, \ldots, S_{V}\right]
$$

such that

$$
\sum_{v=1}^{V} S_{v}=S
$$

and then these subblocks are combined to minimize the PAPR in time domain. In time domain the signal $s_{v}$ is oversampled $F$ times which is obtained by taking an IFFT of length $F N$ on signal $X_{v}$ concatenated with $(F-1) N$ zeros. After partitioning the signal and performing the IFFT for each part, then the phase factors $b_{v}=e^{j \phi_{v}}, v=1,2, \ldots, V$ are used to optimize the $S_{v}$. In time domain the OFDM signal can be expressed as:

$$
s^{\prime}(b)=\sum_{v=1}^{V} b_{v} s_{v}
$$

where $s^{\prime}(b)=\left[s_{0}^{\prime}(b), s_{1}^{\prime}(b), \ldots s_{N F-1}^{\prime}(b)\right]^{T}$. The objective is to find the optimum signal $s^{\prime}(b)$ with the lowest PAPR. Notice that here $N=K+L$ which means that there is no change in the length of the input signal after the addition of dummy sequence. The subblock partition type here is based on interleaving which is the best choice for PTS OFDM in terms of computational complexity reduction as compared to adjacent and pseudo-random method, however it gives the least PAPR reduction among them.

Then, the process is continued by choosing the optimization parameter $\tilde{b}$ with the following condition:

$$
\tilde{b}=\arg \min \left(\max _{0 \leq k \leq N F-1}\left|\sum_{v=1}^{V} b_{v} s_{v, k}\right|\right)
$$

After finding the optimum $\tilde{b}$ then the optimum signal $s^{\prime}(b)$ is transmitted to the next block. Then the PAPR of $s^{\prime}(b)$ is checked whether it lies in the range of the PAPR threshold $\left(P A P R_{t h}\right)$. After this additional task, the signal is transmitted otherwise it is returned to the DSI block to generate the dummy sequence again. This process will continue until the PAPR is less than the $P A P R_{t h}$.

Fig. 7 shows the CCDF curves of conventional PTS and DSI-PTS techniques. We assume here that the number of dummy sequence insertion $(L)$ is 55 which bears no significant 
effect on the transmission efficiency $(T E=100 \%)$. These results are obtained after 10 iteration (I). It can be observed that the PAPR reduction of our proposed PTS scheme outperforms the conventional PTS scheme with an improvement by 2 and $1 \mathrm{~dB}$ respectively at $C C D F=0.01 \%$, when $V=2,4$ respectively. Even though this reduction seems minor the complexity according to Table 1 is reduced significantly.

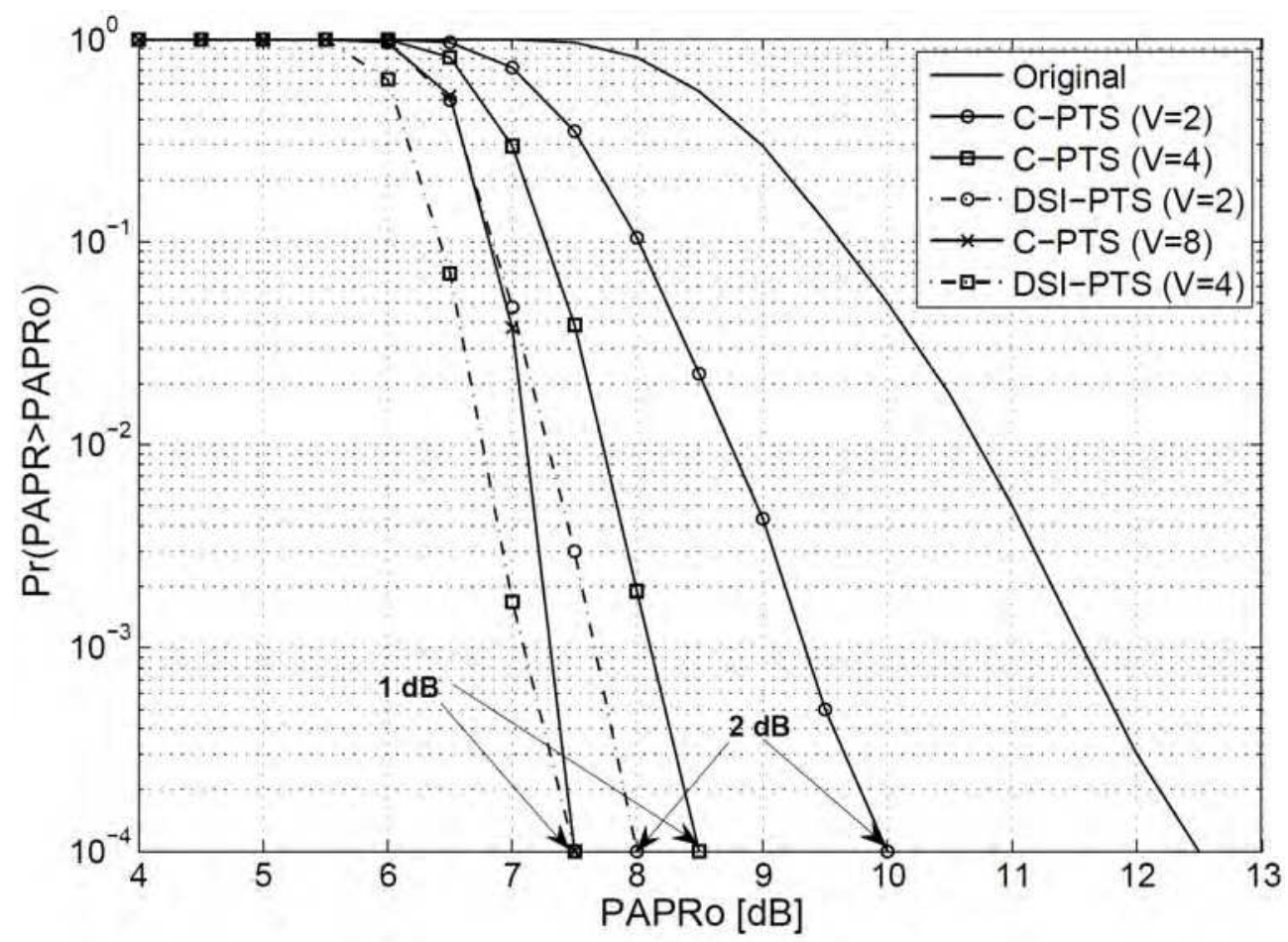

Fig. 7. CCDF of the PAPR of conventional PTS and DSI-PTS technique (L=55, $I=10)$.

Fig. 8 shows the result for different length of dummy sequence. As discussed earlier the maximum length of dummy sequence that can be applied is 55 and this figure shows that with this length the reduction obtained is slightly better than when is 30 . It is observed that the reductions of PAPR at $C C D F=0.01 \%$ are $1 \mathrm{~dB}, 1.5 \mathrm{~dB}$ and $2 \mathrm{~dB}$ for dummy length of 5, 30 and 55 respectively.

Fig. 9 shows the effect of different iteration number on the PAPR performance. From this figure maximum PAPR reduction is achieved which is $7 \mathrm{~dB}$ at $C C D F=0.01 \%$ at 100 iterations with $L=55$. But increasing the number of iterations will reduce the data rate. 


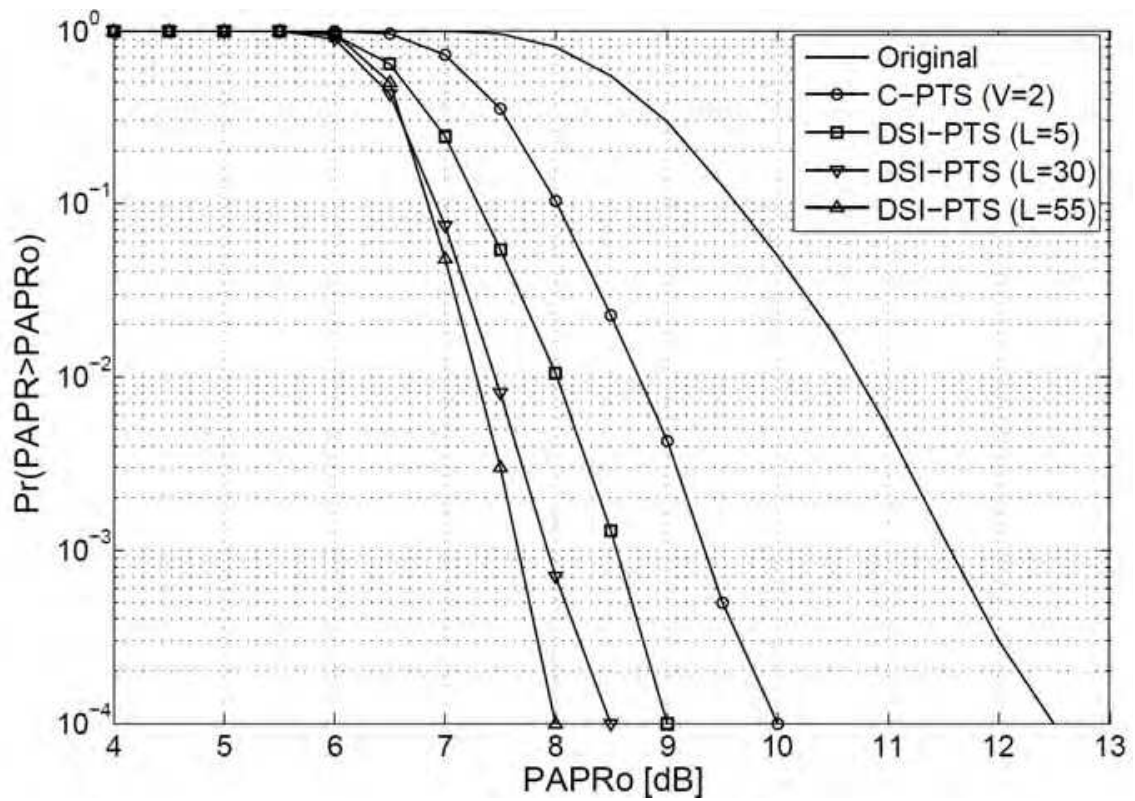

Fig. 8. CCDF of PAPR of DSI-PTS technique for different length of dummy sequence when $\mathrm{I}=10$.

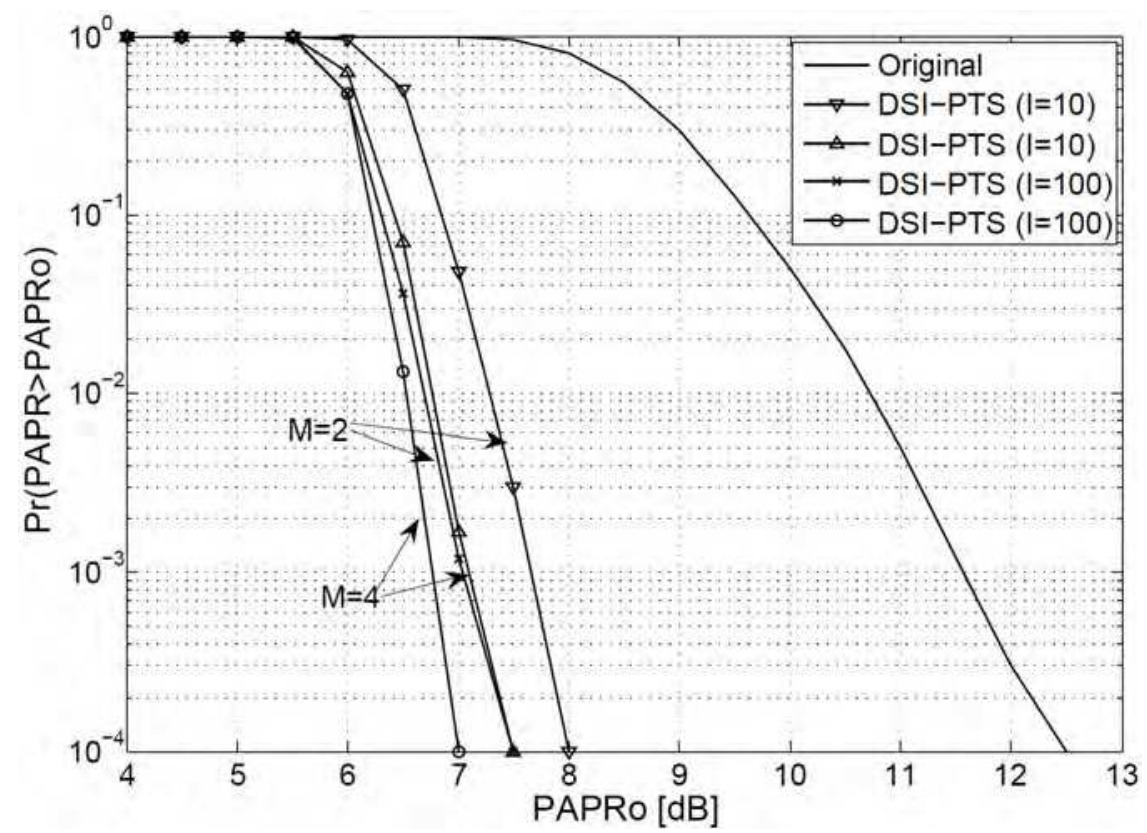

Fig. 9. CCDF of PAPR of DSI-PTS technique for different number of iterations when the $\mathrm{L}=55$ 
There is about $0.5 \mathrm{~dB}$ improvement in PAPR reduction when the number of iteration is 100 compared to 10 iteration for both cases of $V=2,4$ as shown in Fig. 9.

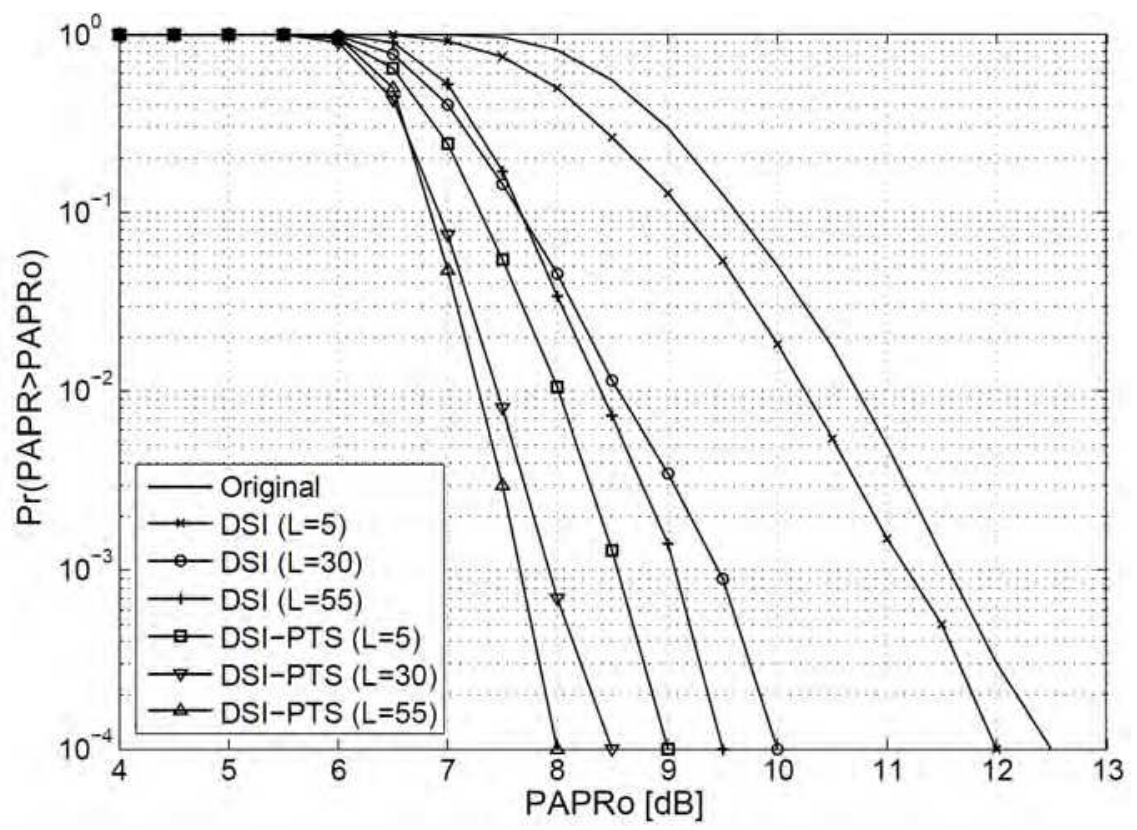

Fig. 10. CCDF of PAPR of DSI-PTS technique compared to DSI when the number of iterations is 10 and $V=2$.

Fig. 10 demonstrates the PAPR reduction capacity in DSI and DSI-PTS techniques. It should also be highlighted on that. The DSI-PTS technique offers about $1.5 \mathrm{~dB}$ further reduction in PAPR compared to DSI when the number of dummy sequence $L=55$ and $V=2$.

\subsection{Dummy Sequence Insertion with Enhanced Partial Transmit Sequence (DSI-EPTS)}

The block diagram of this technique is shown in Fig. 11. Here as in DSI described previously, the complex valued dummy signals are first generated and then added to the vector of data subcarriers. The new vector in the frequency domain is then constructed from $K$-data and $L$-dummy subcarriers, respectively. $L$ can be any number less than $K$. The new vector $U$ is given by:

$$
U=\left[X_{k}, W_{l}\right]
$$

where $X_{k}=\left[X_{k, 0}, X_{k, 1}, \ldots, X_{k, N-L-1}\right], k=1,2, \ldots, K$ is the data subcarrier vector and $W_{l}=\left[W_{l, 0}, W_{l, 1}, \ldots, W_{l, L-1}\right], \quad l=1,2, \ldots, L$ is the dummy signals vector.

After generation of the optimum OFDM signal, PAPR is checked with the acceptable threshold that has been predefined before. If the PAPR value is less than the threshold then the OFDM signal will be transmitted otherwise the dummy sequence is generated again as 
shown by the feedback loop in Fig. 11. This process is one iteration. The number of iterations can be increased to achieve the desired PAPR $\left(P A P R_{t h}\right)$ reduction but the processing time will also increase likewise and cause the system performance to drop. From the block diagram in Fig. 11, $X$ is the input signal with length $N$. After that dummy sequence is added which causes an increase in the IFFT length.

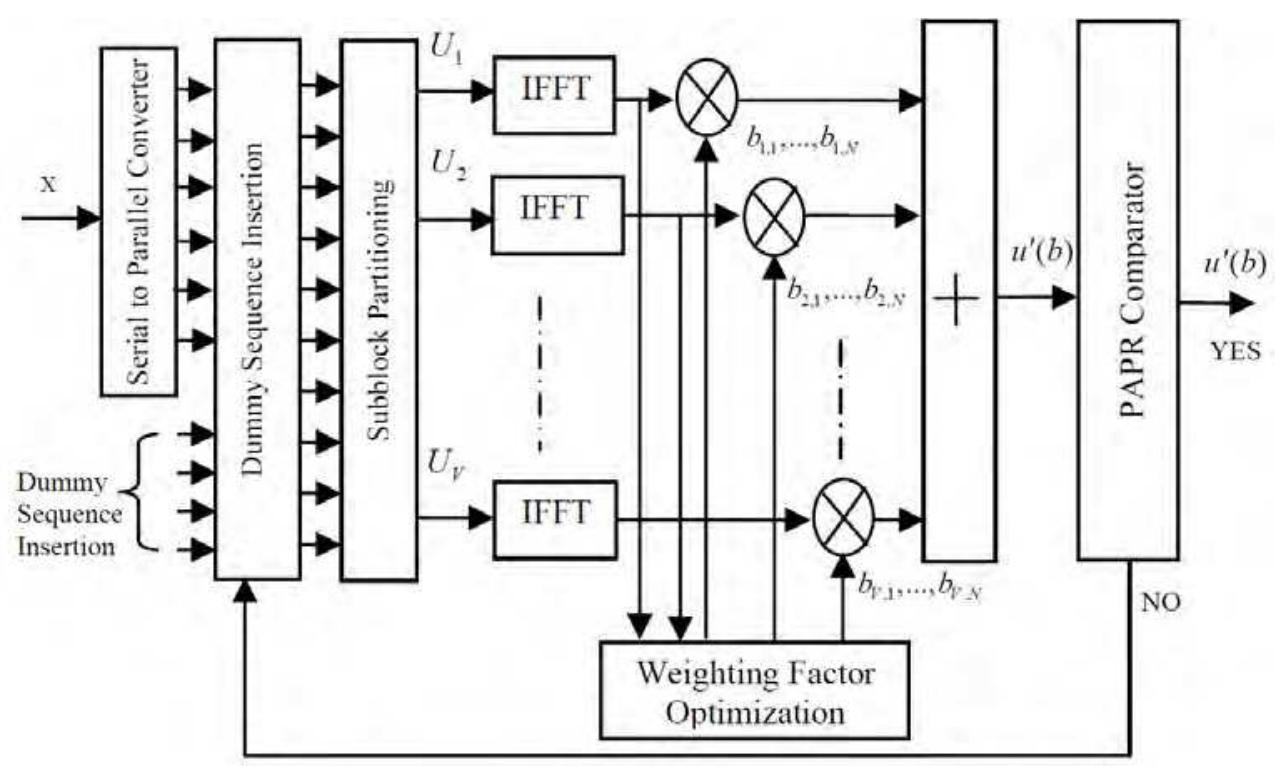

Fig. 11. Block diagram of the proposed DSI-EPTS scheme

The same procedure similar to the one discussed in section 3.5 for DSI-PTS scheme is performed here except the phase sequence is taken from the EPTS scheme discussed earlier in section 3.5.

\subsubsection{Computational complexity}

The total complexity of the C-PTS with oversampling factor $F=1$, is given by (Baxley and Zhou, 2007):

$$
T_{C-P T S}=3 V N / 2 \log N+2 V W^{V-1} N
$$

Whereas for the Enhanced PTS this value is:

$$
T_{E P T S}=3 / 4 V N \log N+P V N
$$

where $P$ is the number of iterations and $V$ is the number of subblocks.

In (Varahram, et al. 2010), the complexity is calculated only for IFFT section, but here we require the total complexity. Hence the total complexity for the DSI-PTS method is given by: 


$$
T_{D S I-P T S}=3 / 4 V N \log N+2 V N W^{V-1}+Q L
$$

The total complexity of DSI-EPTS is given by;

$$
T_{D S I-E P T S}=3 / 4 V N \log N+P V N+Q L
$$

where $Q$ is the number of iterations for the DSI loop.

It can be observed that (22) and (23) consist of two parts; the first part is actually the complexity of the IFFT itself and the second part is the complexity of the searching algorithm. Most of the papers did not consider the second part which causes wrong calculation of the complexity. It should be noted that the number of IFFT in (24) and (25) is halved which basically is concluded from the simulation results. From the simulation results given in the following section the PAPR performance of the proposed method when the number of IFFT is half of the C-PTS, is almost the same. This is shown for different number of subblocks which proves that in the DSI-EPTS the number of IFFT is halved compared to the C-PTS but gives the same PAPR performance.

\begin{tabular}{|c|c|c|c|c|}
\hline \hline \multirow{2}{*}{ Total Complexity } & $\begin{array}{c}\text { No. of } \\
\text { Subblocks }\end{array}$ & C-PTS & DSI-PTS & CCRR \\
\cline { 2 - 5 } & $\mathrm{V}=4$ & 60416 & 46760 & $22.6 \%$ \\
\hline
\end{tabular}

Table 1. Computational Complexity of the DSI-PTS and the conventional PTS when $N=512$ and $W=2, Q=3, L=56$

\begin{tabular}{|c|c|c|c|c|c|c|}
\hline \hline & \multirow{2}{*}{$\begin{array}{c}\text { No. of } \\
\text { Subblocks }\end{array}$} & \multirow{2}{*}{ C-PTS } & \multicolumn{2}{|c|}{ DSI-EPTS } & \multicolumn{2}{c|}{ CCRR (\%) } \\
\cline { 4 - 7 } & & $\mathrm{D}=1$ & $\mathrm{D}=2$ & $\mathrm{D}=1$ & $\mathrm{D}=2$ \\
\hline \multirow{2}{*}{$\begin{array}{c}\text { Total } \\
\text { Complexity }\end{array}$} & $\mathrm{V}=4$ & 60416 & 30376 & 46760 & 49.7 & 22.6 \\
\cline { 2 - 7 } & $\mathrm{V}=8$ & 1103872 & 552104 & 1076392 & 49.6 & 2.7 \\
\hline
\end{tabular}

Table 2. Computational Complexity of the DSI-EPTS and the conventional PTS when $N=512$ and $W=2, Q=3, L=56$

The computational complexity reduction ratio (CCRR) of the proposed technique over the C-PTS is defined as (Baxley and Zhou, 2007):

$$
\text { CCRR }=\left(1-\frac{\text { Complexity of the DSI }- \text { EPTS }}{\text { Complexity of the } C-P T S}\right) \times 100 \%
$$


Table 1 presents the computational complexity of C-PTS and DSI-PTS, for $N=512$ and $W=2$.

Table 2 presents the computational complexity of C-PTS and proposed DSI-EPTS, for the same value of $N$ and $W$, while $\mathrm{D}$ is the coefficient that can be specified based on the PAPR reduction and complexity according to equation (12).

It is clear from Table 2, that CCRR is improved for both $V=4$ and $V=8$. It should be noted that when $D$ increases, the complexity reduction becomes less while PAPR performance improves, as shown in the simulations.

\subsubsection{Side information}

The other important factor in studying the PAPR reduction method is the side information which has to be transmitted to the receiver to extract the original signal. One method is that the side information can be transmitted in a separate channel but this comes at the expense of spectrum efficiency degradation.

The number of required side information bits in C-PTS is

$$
\left\lfloor\log _{2} W^{V-1}\right\rfloor
$$

where $W$ is the number of allowed phase factors and the sign $L\rfloor$ indicates the floor of y. In DSI-EPTS, the side information can be allocated in the dummy signals and therefore does not have impact on spectrum efficinecy and data rate loss; however, the only drawback of this method is that, because of the increase in the phase sequence matrix, higher memory space is required.

\subsubsection{System performance}

In C-PTS, even though an OFDM signal does not experience distortion the signal after power amplifier could exhibit distortions if PAPR is higher than the expected value. In this case the power amplifier should back off which degrades the efficiency of the system. In DSI-EPTS, the addition of dummy sequences causes the transmission efficiency to change as follows:

$$
T E=\frac{K}{K+L} \times 100[\%]
$$

where $K$ is the length of subcarriers and $L$ is the length of dummy sequences. In actual applications where the cost of the system is the main issue, the other block also have to be considered, the digital predistortion (DPD) (Varahram and Atlasbaf, 2005), (Varahram, et al. 2005). By applying DPD technique, it is possible to increase the linearity of the power amplifier and as a result, higher peak signals can be transmitted by the power amplifier and the performance of the PAPR can be improved. This also increases the efficiency of the power amplifiers and decreases the cost of the system.

Fig. 12 shows the CCDF comparison of PAPR of DSI-EPTS with C-PTS. It is clear that by applying the DSI-EPTS when $\mathrm{D}=2$, the PAPR performance is more superior over that of CPTS for both $V=4$ and $V=8$ respectively. But PAPR reduction when $D=1$ is almost the same as $\mathrm{C}$-PTS for $\mathrm{V}=4$ and $\mathrm{V}=8$ respectively. 


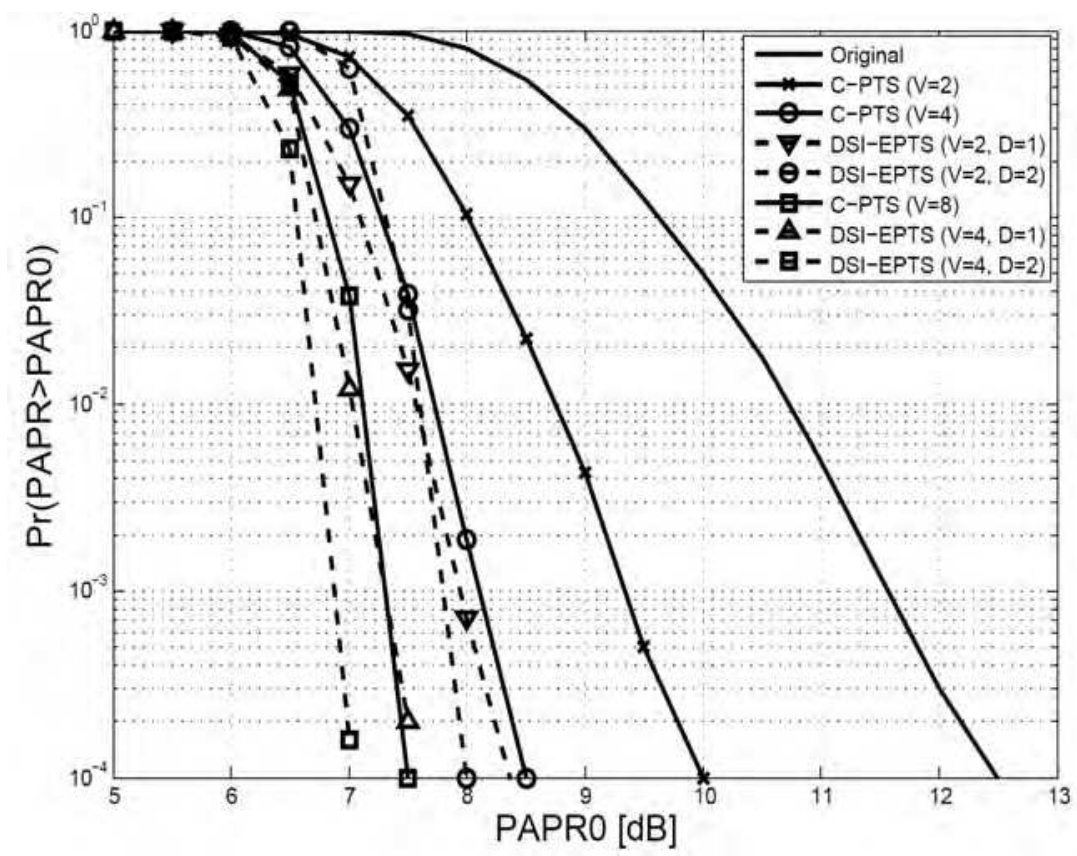

Fig. 12. CCDF comparison of PAPR of the DSI-EPTS and C-PTS

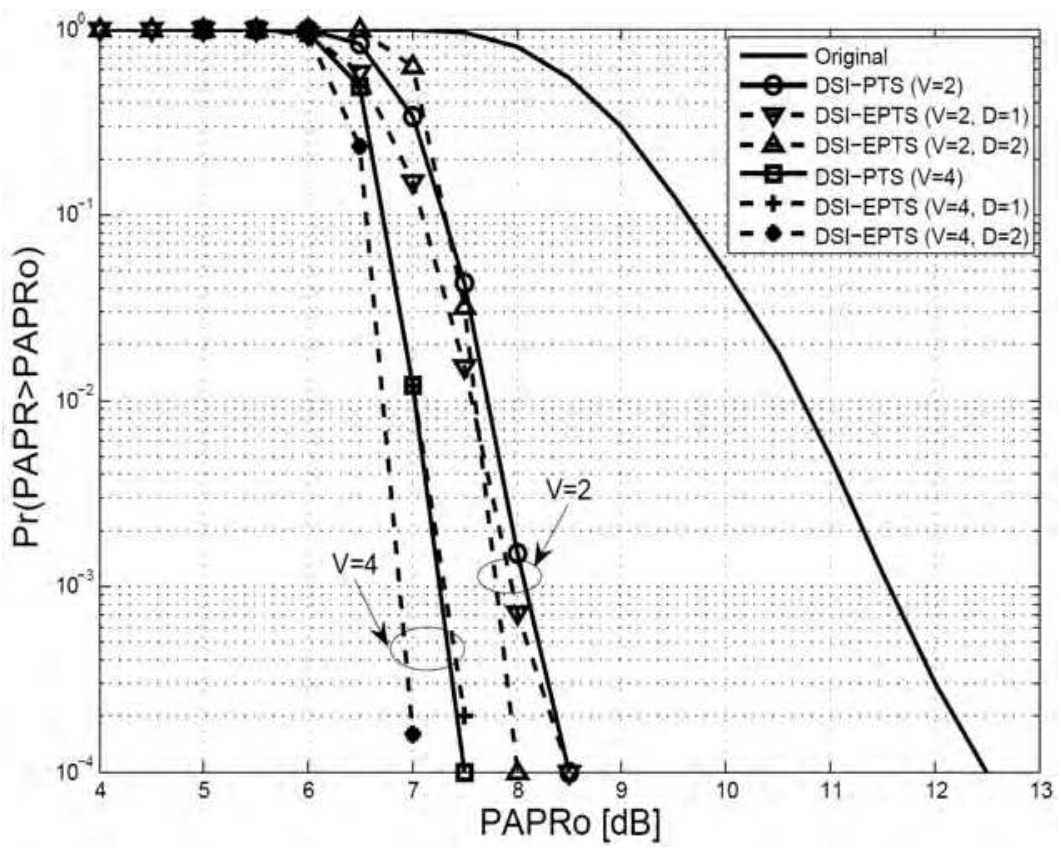

Fig. 13. CCDF comparison of PAPR of the DSI-EPTS and DSI-PTS when L=56 
The highest PAPR reduction is achieved when $\mathrm{D}=2$ and $\mathrm{V}=4$. From table 2, the complexity reduction is minimum when $\mathrm{D}=2$. There is always a trade off between PAPR reduction performance and complexity reduction.

Fig. 13 shows the CCDF comparison of PAPR of the DSI-EPTS and DSI-PTS when L=56. The results are shown for $\mathrm{V}=2$ and $\mathrm{V}=4$. The CCDF results show that PAPR of the DSI-EPTS outperforms DSI-PTS for both $\mathrm{V}=2$ and $\mathrm{V}=4$ respectively.

Fig. 14 shows a comparison of Bit Error Rate (BER) performance of the conventional PTS and the proposed EPTS and DSI-EPTS method in Additive White Gaussian Noise (AWGN) channels. The length of dummy sequence and iterations is $L=56$. From this figure, we can see that the BER is slightly increased with DSI-EPTS method compared to conventional PTS, but PAPR is much improved according to the result of Fig. 13. The performance of the system shows improvement at the cost of BER.

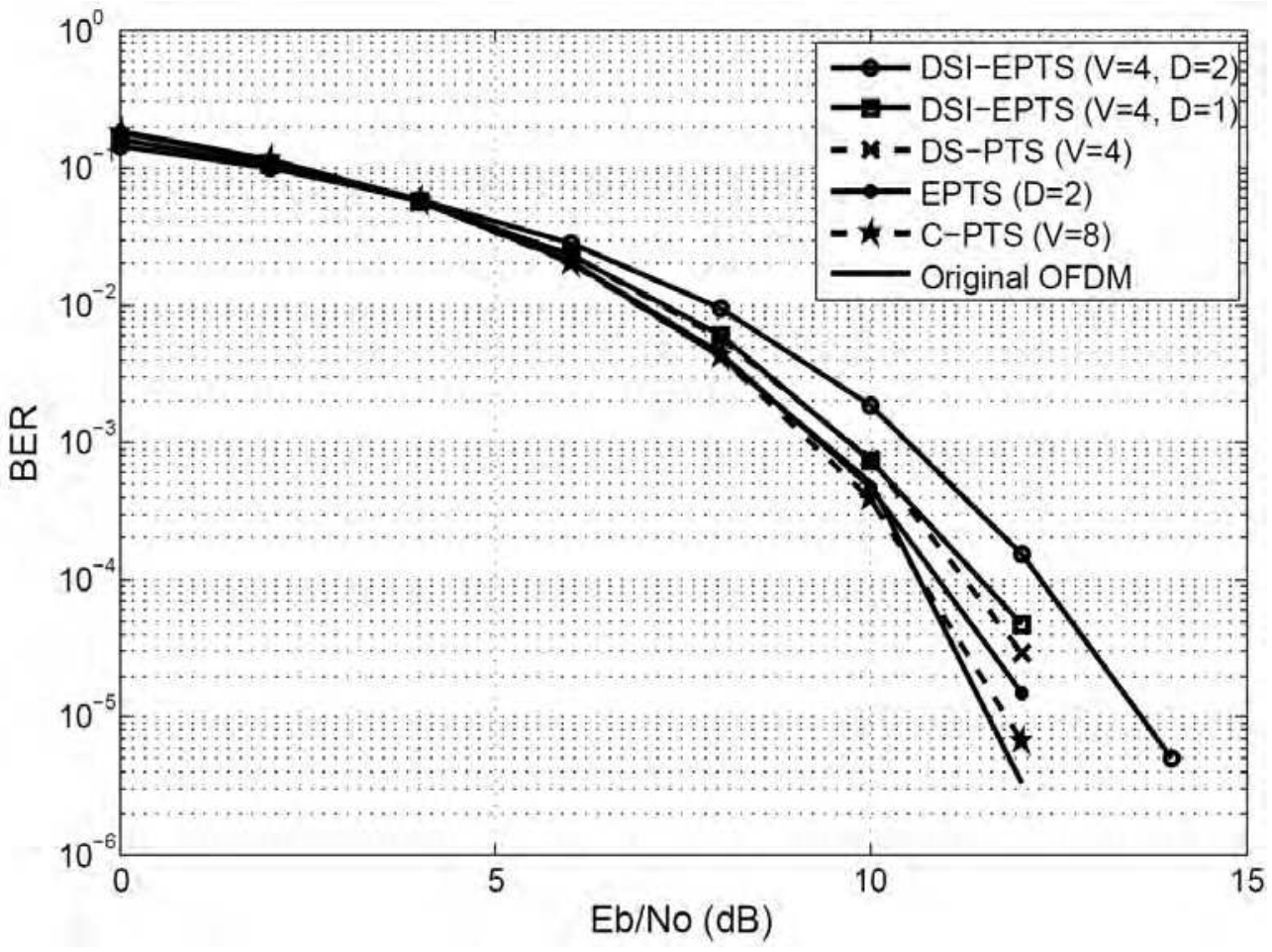

Fig. 14. Comparison of BER performance of the conventional PTS and DSI-PTS technique in AWGN channels. 


\section{Conclusion}

In this chapter we have studied and discussed several PAPR redcution techniques. Their advantages and disadvantages have been analyzed and by performing the simulation results, the PAPR performance of those techniques have been compared. Also the complexity of each technique has been computed and finally compared. These PAPR techniques is ideal for the latest wireless communications systems such as WiMAX and long term evolution (LTE).

\section{Acknowledgment}

This work was supported by Universiti Putra Malaysia under the Research University Grant Scheme (No. 0501090724RU).

\section{References}

Al-Azoo W. F., Ali B. M, Khatun S., Bilfagih S. M, and Noordin N. K., "Addition of Gaussian random signals for peak to average power ration reduction in OFDM systems," IEEE ICCCE'08 International conference, PP. 1344-1347, 2008.

Bauml R. W., Fischer R. F. H. and Huber J. B., "Reducing the peak-to-average power ratio of multicarrier modulationby selected mapping," Electron. Lett., vol. 32, pp. 2056-2057, 1996.

Bauml v, Fischer R. F. H. and Huber J. B., "Reducing the peak-to-average power ratio of multicarrier modulationby selected mapping," Electron. Lett., vol. 32, pp. 2056-2057, 1996.

Baxley R. J. and Zhou T., "Comparing slected mapping and partial transmit sequence for PAPR reduction", IEEE Trans. Broadcast., vol. 53, no. 4, pp.797-803, December 2007.

Cimini L. J. and Sollenberger N. R., "Peak-to-average power ratio reduction of an OFDM signal using partial transmit sequences," IEEE Commun. Lett., vol. 4, no. 3, pp. 8688, March. 2000.

Han S. H. and Lee J. H., "An overview of peak-to-average power ratio reduction techniques for multicarrier transmission," IEEE Wireless Commun., vol. 12, no. 2, pp. 56-65, Apr. 2005.

Hao M. and Liaw C., "A companding technique for PAPR reduction of OFDM systems," IEICE Trans. Commun., vol. E91-B, pp. 935-938, 2008.

Foomooljareon P. and Fernando W. A. C., "Input sequence envelope scaling in PAPR reduction of OFDM," in 2002.

Jayalath v and Tellambura C.. (2000, Reducing the peak-to-average power ratio of orthogonal frequencydivision multiplexing signal through bit or symbol interleaving. Electron. Lett. 36(13), pp. 1161-1163.

Krongold B. S. and Jones D. L., "PAR reduction in OFDM via active constellation extension," IEEE Trans. Broadcast., vol. 49, 2003.

Kwon U., Kim D. and Im G., "Amplitude clipping and iterative reconstruction of MIMOOFDM signals with optimum equalization," IEEE Transactions on Wireless Communications, vol. 8, pp. 268-277, 2009. 
Mohammady S., Varahram P., Sidek R. M., Hamidon M. N. and Sulaiman N., “Efficiency improvement in microwave power amplifiers by using Complex Gain Predistortion technique," IEICE Electronics Express (ELEX), vol. 7, no. 23, pp.17211727, Oct. 2010.

Muller S. H. and Huber J. B., "A novel peak power reduction scheme for OFDM," in Personal, Indoor and Mobile Radio Communications, 1997.'Waves of the Year 2000'. PIMRC'97., the 8th IEEE International Symposium on, 1997.

Nikookar H. and Lidsheim K. S., "Random phase updating algorithm for OFDM transmission with low PAPR," IEEE Trans. Broadcast., vol. 48, pp. 123-128, 2002.

Qian H., "Power Efficiency Improvements for Wireless Transmissions," Power Efficiency Improvements for Wireless Transmissions, 2005.

Ryu H. G., Lee J.E. and Park J.S., “Dummy sequence insertion (DSI) for PAPR reduction in the OFDM communication system," IEEE Trans. Consumer Electr., vol. 50, no. 1, pp. 89-94, Feb. 2004

Tellado J., Multicarrier Modulation with Low PAR: Applications to DSL and Wireless. Kluwer Academic Pub, 2000.

Tan C. E. and Wassell I. J., "Data bearing peak reduction carriers for OFDM systems," Information, Communications and Signal Processing, 2003 and the Fourth Pacific Rim Conference on Multimedia. Proceedings of the 2003 Joint Conference of the Fourth International Conference on, vol. 2; 2, pp. 854-858 vol.2, 2003.

Van Nee R. and De Wild A., "Reducing the peak-to-average power ratio of OFDM," in 48th IEEE Vehicular Technology Conference, 1998. VTC 98, 1998.

Varahram P., Mohammady S., Hamidon M. N., Sidek R. M. and Khatun S., “Digital Predistortion Technique for Compensating Memory Effects of Power Amplifiers in Wideband Applications", Journal of Electrical Engineering, vol. 60, no. 3, 2009.

Varahram P., Mohammady S., Hamidon M. N., Sidek R. M. and Khatun S., "Power amplifiers linearization based on digital predistortion with memory effects used in CDMA applicationsm," 18th European Conference on Circuit Theory and Design ECCTD, pp. $488-491,2007$.

Varahram P., Atlasbaf Z., "Adaptive digital predistortion for high power amplifiers with memory effects," Asia-Pacific Microwave Conference Proceedings, APMC. 3(1606627), 2005.

Varahram P., Azzo W. A., Ali B. M., “A Low Complexity Partial Transmit Sequence Scheme by Use of Dummy Signals for PAPR Reduction in OFDM Systems," IEEE Trans. Consumer Electron, vol. 56, no. 4, pp. 2416-2420, Nov. 2010.

Varahram P., Atlasbaf Z., Heydarian N., "Adaptive digital predistortion for power amplifiers used in CDMA applications," Asia-Pacific Conference on Applied Electromagnetics, APACE Proceedings, (1607810):215-21, 2005.

Vijayarangan V. and Sukanesh D., "AN OVERVIEW OF TECHNIQUES FOR REDUCING PEAK TO AVERAGE POWER RATIO AND ITS SELECTION CRITERIA FOR ORTHOGONAL FREQUENCY DIVISION MULTIPLEXING RADIO SYSTEMS," pp. 25, 2009. 
Wilkison T. A. and Jones A. E., "Minimazation of the peak to mean envelope power ratio of multicarrier transmission schemes by block coding," in IEEE Vehicular Technology Conference, 1995. 


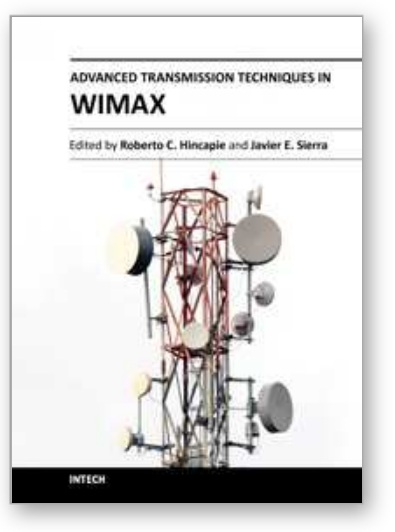

\author{
Advanced Transmission Techniques in WiMAX \\ Edited by Dr. Roberto Hincapie
}

ISBN 978-953-307-965-3

Hard cover, 336 pages

Publisher InTech

Published online 18, January, 2012

Published in print edition January, 2012

This book has been prepared to present the state of the art on WiMAX Technology. The focus of the book is the physical layer, and it collects the contributions of many important researchers around the world. So many different works on WiMAX show the great worldwide importance of WiMAX as a wireless broadband access technology. This book is intended for readers interested in the transmission process under WiMAX. All chapters include both theoretical and technical information, which provides an in-depth review of the most recent advances in the field, for engineers and researchers, and other readers interested in WiMAX.

\title{
How to reference
}

In order to correctly reference this scholarly work, feel free to copy and paste the following:

Pooria Varahram and Borhanuddin Mohd Ali (2012). Peak-to-Average Power Ratio Reduction in Orthogonal Frequency Division Multiplexing Systems, Advanced Transmission Techniques in WiMAX, Dr. Roberto Hincapie (Ed.), ISBN: 978-953-307-965-3, InTech, Available from:

http://www.intechopen.com/books/advanced-transmission-techniques-in-wimax/peak-to-average-power-ratioreduction-in-orthogonal-frequency-division-multiplexing-systems

\section{INTECH}

open science | open minds

\section{InTech Europe}

University Campus STeP Ri Slavka Krautzeka 83/A 51000 Rijeka, Croatia Phone: +385 (51) 770447

Fax: +385 (51) 686166 www.intechopen.com

\section{InTech China}

Unit 405, Office Block, Hotel Equatorial Shanghai No.65, Yan An Road (West), Shanghai, 200040, China 中国上海市延安西路65号上海国际贵都大饭店办公楼405单元 Phone: +86-21-62489820

Fax: +86-21-62489821 
(C) 2012 The Author(s). Licensee IntechOpen. This is an open access article distributed under the terms of the Creative Commons Attribution 3.0 License, which permits unrestricted use, distribution, and reproduction in any medium, provided the original work is properly cited. 